\title{
Desenvolvimento e Validação Preliminar de uma Escala Multidimensional de Satisfação de Vida para Adolescentes ${ }^{1}$
}

\author{
Joice Dickel Segabinazi ${ }^{2}$ \\ Universidade Federal do Rio Grande do Sul \\ Claudia Hofheinz Giacomoni \\ Universidade Federal do Rio Grande do Sul \\ Ana Cristina Garcia Dias \\ Universidade Federal de Santa Maria \\ Marco Antônio Pereira Teixeira \\ Universidade Federal do Rio Grande do Sul \\ Denis Altieri de Oliveira Moraes \\ Universidade Federal de Santa Maria
}

\begin{abstract}
RESUMO - O objetivo deste estudo foi desenvolver uma Escala Multidimensional de Satisfação de Vida para Adolescentes (ESMVA). Participaram 425 adolescentes (224 meninos e 201 meninas), com idade média de 16,1 anos (DP=1,2). Os itens foram selecionados por meio de procedimentos de análise de componentes principais, e a versão final da ESMVA contou com 52 itens, distribuídos em sete componentes: Família, Self, Escola, Self Comparado, Não-violência, Auto-eficácia e Amizade. O resultado mostrou uma estrutura que explicou $54 \%$ da variância. A análise da consistência interna, medida pelos valores de alfa de Cronbach, foram adequadas para cada uma das subescalas, assim como para a escala total $(\alpha=0,93)$. Evidências de validade foram obtidas também por meio de correlações com uma medida de auto-estima.
\end{abstract}

Palavras-chave: bem-estar subjetivo; escala de satisfação de vida; adolescentes.

\section{Development and Preliminary Validation of a Multidimensional Life Satisfaction Scale for Adolescents}

\begin{abstract}
The purpose of this study was to develop a Multidimensional Life Satisfaction Scale for Adolescents (MLSSA). Participants were 425 adolescents (224 boys and 201 girls) with a mean age of 16,1 years $(\mathrm{SD}=1,2)$ attending schools in the city of Santa Maria/RS. Items selection occurred on basis of the results from principal components analysis, and the final form of the MLSSA included 52 items, distributed over 7 factors: Family, Self, Compared Self, School, Non-violence, Selfefficacy and Friendship. The seven components explained 54\% of the total variance. The coefficients of internal consistency were satisfactory for each subscale, as well as for the total scale $(\alpha=0,93)$. Evidences of validity were also obtained through a correlational analysis with a measure of Self-esteem.
\end{abstract}

Keywords: subjective well-being; life satisfaction scale; adolescents.

Nas últimas décadas, a Psicologia tem se interessado cada vez mais pelo desenvolvimento psicológico saudável nas diferentes etapas do ciclo vital, perspectiva que tem sido chamada de Psicologia Positiva (Seligman \& Csikszentmihalyi, 2000). No entanto, observa-se que apenas recentemente essa abordagem passou a ser aplicada aos estudos sobre a adolescência, buscando fatores antecedentes da satisfação com a vida em adolescentes (Ben-Zur, 2003; Hunter \& Csikszentmihalyi, 2003; Suldo \& Huebner, 2006).

$\mathrm{O}$ bem-estar subjetivo (BES) se insere nessa perspectiva positiva e seu conceito inclui as avaliações afetivas e cognitivas que o indivíduo faz a respeito de sua existência. Enquanto o componente afetivo abrange afetos positivos e

1 Agradecemos aos integrantes do LEMAP - UFSM (Laboratório de Estudo Medidas e Avaliação em Psicologia) pela participação na fase de coleta, digitação e análise dos dados. Apoio Financeiro: FIPE - Fundo de Incentivo à Pesquisa da Universidade Federal de Santa Maria (UFSM).

2 Endereço para correspondência: Rua Ramiro Barcelos, 2600 sala 120 Bairro Santana, CEP 90035-003 - Porto Alegre, RS - Brasil, Telefone: (51)3308.5352 Fax: (51) 3308.5473 E-mail: jsegabinazi@gmail.com negativos vivenciados pelo indivíduo, a satisfação de vida corresponde à avaliação cognitiva de sua vida, baseada em padrões selecionados pela própria pessoa (Diener, Suh, Lucas, \& Smith, 1999). Esse elemento pode ser avaliado globalmente, considerando a vida como um todo, e a partir de domínios específicos, por exemplo, o trabalho, a família e o lazer. Diener (1984) salienta a importância de se estimar a satisfação de vida a partir de domínios ou dimensões, pois são as dimensões mais relevantes eleitas pelas pessoas as que mais influenciam o BES.

Encontram-se na literatura modelos multidimensionais de avaliação da satisfação de vida na adolescência. Huebner, Drane e Valois (2000) propuseram que a satisfação poderia ser avaliada globalmente e também em cinco domínios teoricamente relevantes: família, amigos, escola, Self e ambiente onde vivem. Na pesquisa, os adolescentes relataram níveis positivos de satisfação global e nas dimensões específicas. Os autores não encontraram diferenças significativas para sexo, raça, série e idade na avaliação da satisfação de vida global. Encontraram-se diferenças de gênero entre a satisfação com 
as experiências escolares, dentre as quais as meninas apontaram maior satisfação. Os autores também observaram diferenças significativas para amizade, tendo as meninas maiores níveis de satisfação em seus relacionamentos com os pares.

A partir da construção deste modelo multidimensional e de propostas de avaliação, pesquisas internacionais têm buscado identificar aspectos associados ao bem-estar e à satisfação de vida na adolescência. Podem-se observar relações destas variáveis com recursos pessoais dos adolescentes e a satisfação de vida de seus pais (Ben-Zur, 2003), auto-estima, lócus de controle e expectativas para o futuro (Hunter \& Csikszentmihalyi, 2003). Ademais, investigações realizadas em outros países têm apresentado correlações significativas entre auto-estima e satisfação de vida em crianças (Huebner, 1994; Huebner, Gilman, \& Laughlin, 1999; Terry \& Huebner, 1995). No Brasil, embora utilizando diferentes instrumentos de avaliação, os estudos têm mostrado que, de um modo geral, os jovens apresentam bons níveis de satisfação, o que corrobora os achados da literatura internacional (Arteche \& Bandeira, 2003; Assis, Avanci, Silva, Malaquias, Santos \& Oliveira, 2003; Wagner, Ribeiro, Arteche \& Bornholdt, 1999).

Uma das dificuldades na pesquisa sobre satisfação de vida com adolescentes no Brasil é a falta de instrumental adequado. Existem escalas para avaliar satisfação de vida em crianças (Giacomoni \& Hutz, 2008) e adultos (Albuquerque \& Troccóli, 2004); porém, não foram localizados instrumentos específicos para adolescentes. Os estudos internacionais que utilizaram a Escala de Satisfação de Vida Multidimensional de Huebner (1994) sugerem que crianças e adolescentes diferenciam entre os domínios de satisfação de vida (Gilman, Huebner \& Laughlin, 2000; Huebner, 1998a, 1998b; Huebner, Laughlin, Ash, \& Gilman, 1998; Huebner \& Gilman, 2002). No Brasil, os domínios da Escala Multidimensional de Satisfação de Vida para Crianças (Giacomoni \& Hutz, 2008) não foram testados em adolescentes, porém a medida apresenta um conjunto de dimensões possivelmente aplicáveis a essa população. Nesse instrumento, a partir de um estudo qualitativo prévio realizado com crianças e da análise fatorial dos itens elaborados, foram identificados seis fatores principais para a satisfação de vida: Família, Amigos, Escola, Self, Não-violência e Self Comparado.

No modelo de satisfação de vida proposto por Giacomoni e Hutz (2008), o domínio Self Comparado relaciona-se às teorias de comparação social, segundo a qual, quando os indivíduos avaliam suas vidas, são realizadas comparações com vários padrões, que podem ser outras pessoas, condições passadas, aspirações, necessidades ou metas (Michalos, 1985). A influência do modelo de metas também é ressaltada nessa perspectiva multidimensional. A idéia é que as metas pelas quais as pessoas se empenham, as formas pelas quais elas tentam atingir seus objetivos e os sucessos alcançados com essas metas ocupam um lugar importante na avaliação da satisfação de vida (Cantor, 1994; Cantor \& Sanderson, 1999; Diener \& Lucas, 1999, Emmons, 1986).

Uma vez que o progresso nos estudos sobre bem-estar subjetivo e satisfação de vida em adolescentes no nosso país depende do desenvolvimento de medidas especialmente construídas para essa população, o objetivo deste trabalho é apresentar estudos de desenvolvimento e validação preliminar de uma Escala Multidimensional de Satisfação de
Vida para Adolescentes. Especificamente, buscou-se: (a) identificar dimensões distintas e psicologicamente significativas relacionadas à satisfação de vida em adolescentes; (b) examinar as características psicométricas do instrumento construído; (c) avaliar o nível de satisfação dos adolescentes com relação aos domínios específicos de suas vidas; (d) verificar possíveis diferenças entre sexo, faixa etária e tipo de escola nos domínios identificados; (e) investigar evidências de validade da escala a partir da correlação desta com uma medida critério de auto-estima.

\section{Método}

\section{Identificação das dimensões relacionadas à satisfação de vida em adolescentes}

O primeiro passo na elaboração do instrumento foi definir as dimensões de qualidade de vida que seriam abarcadas na escala. Para tanto, utilizou-se como base um estudo qualitativo que entrevistou 95 adolescentes sobre o conceito de felicidade e suas características que identificou onze categorias (Giacomoni e cols., 2005). Seis dessas categorias foram similares aos fatores da Escala Multidimensional de Satisfação de Vida Infantil - EMSVI (Giacomoni \& Hutz, 2008), desenvolvida previamente para crianças, a saber, Self, Self Comparado, Não-violência, Família, Amizade, Escola, e outras cinco categorias foram específicas para os adolescentes: Altruísmo, Autonomia, Lazer, Saúde e Satisfação das Necessidades Materiais e de Desejo (Giacomoni, e cols., 2005).

Além de aproveitaram-se itens da EMSVI, utilizaram-se as definições das categorias desenvolvidas no estudo de Giacomoni e cols. (2005) para a elaboração dos itens que compuseram a EMSVA. O conjunto de itens proposto foi apresentado a dois psicólogos, um especialista na área de avaliação e construção de instrumentos e outro com experiência em estudos sobre adolescência, para que fosse avaliada a pertinência dos itens em cada dimensão da escala. Foram mantidos apenas os itens avaliados, por ambos os juízes, como adequados para cada dimensão. Alterações foram feitas em quatro itens como sugestão de um dos avaliadores.

Para verificar a compreensão dos itens propostos, os instrumentos foram aplicados em dois grupos de três adolescentes. Foi solicitado a cada participante que respondesse a escala individualmente. Ao terminarem, foi realizada uma discussão em grupo, na qual foi solicitado aos adolescentes que apontassem suas dificuldades em relação às instruções e aos termos presentes nos itens da escala. A análise dos dados colhidos levou à modificação de termos em três itens. Chegou-se assim a uma versão com 84 itens. A instrução fornecida aos adolescentes participantes foi a seguinte: "Gostaríamos de saber o que você pensa sobre a sua vida e coisas que fazem parte dela. Por exemplo: como você tem se sentido ultimamente? O que você gosta de fazer? Para cada frase escrita abaixo você deve escolher um dos números que melhor representa o quanto você concorda com o que esta frase diz sobre você". Cada item foi respondido por meio de uma escala de respostas de cinco pontos tipo Likert, variando de (1) nem um pouco a (5) muitíssimo. 


\section{Participantes}

Participaram da pesquisa 425 adolescentes $(52,7 \%$ do sexo masculino), estudantes de escolas privadas (51\%) e públicas do município de Santa Maria/RS. A média de idade foi de 16,1 anos $(\mathrm{DP}=1,2)$, sendo $61,3 \%$ na faixa etária de 14 a 16 anos e o restante entre 17 e 19 anos. A escolha das escolas foi realizada por conveniência.

\section{Instrumentos}

Além de uma folha para identificação de dados pessoais dos participantes, foram aplicadas a Escala Multidimensional de Satisfação de Vida para Adolescentes (EMSVA) e a Escala de Auto-Estima de Rosenberg (1965) em sua versão adaptada para o português por Hutz (2000). A Escala de Auto-Estima de Rosenberg (1965) é uma escala de auto-relato de quatro pontos, que investiga aspectos globais da auto-estima. $\mathrm{Na}$ versão da escala adaptada para o Brasil, um item foi acrescentado, totalizando onze itens e a investigação realizada demonstrou parâmetros psicométricos apropriados (Hutz, 2000). A versão adaptada tem apresentado índices de fidedignidade constantes e acima de 0,80 , sendo aceitável seu uso em pesquisas. Neste estudo, a Escala de Auto-Estima apresentou um índice de consistência interna de 0,82 (alfa de Cronbach).

\section{Procedimentos}

Os instrumentos foram aplicados coletivamente em sala de aula, após a explicação dos objetivos e procedimentos da pesquisa. Termos de consentimento foram obtidos dos pais e dos adolescentes, e os procedimentos foram aprovados pelo Comitê de Ética da Universidade Federal de Santa Maria.

\section{Análise dos dados}

A estrutura dimensional da EMSVA foi verificada por meio de análises de componentes principais. A fidedignidade das escalas foi avaliada através do índice alfa de Cronbach, e os efeitos das variáveis demográficas (sexo, faixa etária e tipo de escola) sobre as dimensões de satisfação de vida foram exploradas por meio de Análise de Variância Multivariada (MANOVA).

\section{Resultados}

\section{Estrutura de componentes e fidedignidade da escala}

O conjunto de 84 itens foi submetido a sucessivas análises de componentes principais com o intuito de identificar os elementos subjacentes ao conjunto de itens, bem como as contribuições de cada item nos mesmos. A escolha desta análise deu-se em consideração ao fato de pesquisas mostrarem que, embora a análise de fatores e a Análise de Componentes
Principais não sejam equivalentes, as diferenças não são importantes quando se trata de grandes conjuntos de dados e grande número de participantes (Dancey \& Reidy, 2006). O resultado da primeira análise indicou que soluções compostas de 6 a 8 componentes eram viáveis e assim foram verificadas soluções contemplando essas possibilidades. A melhor solução foi obtida com a rotação Varimax e possibilitou identificar 8 componentes com significado. Os componentes extraídos confirmaram alguns dos domínios apresentados pelo Modelo de Satisfação de Vida, de Huebner (1994) (Self, Família, Amizade e Escola). Verificou-se também a permanência dos itens relativos aos domínios propostos para a EMSVI por Giacomoni e Hutz (2008) (SelfComparado e Não-violência), e observou-se o surgimento de um novo componente para a EMSVA, que reuniu alguns itens das dimensões Lazer, Autonomia e Satisfação das necessidades materiais e de desejo. Esse fator foi denominado Auto-eficácia pelas características semânticas dos itens agrupados. Os itens com sentido negativo foram revertidos previamente para a análise dos dados.

Análises consecutivas determinaram a eliminação gradual de itens. Optou-se por eliminar o fator Saúde (composto por 5 itens), pois o valor de sua consistência interna foi insatisfatório. Também foram eliminados 11 itens que não apresentaram cargas suficientemente altas (no mínimo 0,30). Outros itens construídos para abarcar os domínios Altruísmo (4 itens), Lazer (5 itens) e Satisfação das Necessidades Materiais e de Desejo (5 itens), que não se agruparam em componentes específicos e permaneceram diluídos em outros componentes foram eliminados. Por fim, outros dois itens foram eliminados, pois seus conteúdos apresentavam similaridade semântica com itens já presentes na escala. Os itens que apresentaram cargas superiores a $0,30 \mathrm{em}$ um segundo fator, além do fator principal, não foram eliminados da escala final. Optou-se por mantê-los devido à sua contribuição teórica para a dimensão, e foram considerados pertencendo à dimensão na qual tiveram a carga mais elevada.

A EMSVA foi submetida a uma nova análise de componentes e finalizou com 52 itens, distribuídos em 7 componentes assim caracterizados: Família, envolve itens descritores de um ambiente familiar saudável, harmônico, afetivo, de relacionamentos satisfatórios; Self, composto por itens que descrevem o adolescente a partir de características positivas, como auto-estima, bom-humor, capacidade de relacionar-se, capacidade de demonstrar afeto, além de indicações de satisfação quanto à diversão; Escola, com itens que descrevem a importância da escola, o ambiente escolar, os relacionamentos interpessoais nesse espaço e nível de satisfação com relação a esse ambiente; Self Comparado, que agrupa itens que se caracterizam por realizar avaliações comparativas do eu ao seu grupo de pares (os itens possuem conteúdos relacionados aos temas lazer, à amizade e à satisfação de desejos e afetos); Não-violência, que inclui itens que envolvem o desejo de não envolvimento em situações de conteúdos associados a comportamentos agressivos como brigas e discussões; Auto-eficácia, que agrupa itens que se caracterizam por avaliações da capacidade de realização e competência no alcance de metas estabelecidas pelo adolescente (os itens possuem conteúdos relacionados a autonomia, lazer, satisfação material e de desejos e Self); Amizade, que caracteriza-se pelos relacionamentos com pares e o nível de 
Tabela 1. Matriz de Componentes da Escala Multidimensional de Satisfação de Vida para Adolescentes

\begin{tabular}{|c|c|c|c|c|c|c|c|}
\hline \multirow{2}{*}{ Dimensão / Item } & \multicolumn{7}{|c|}{ Componente } \\
\hline & 1 & 2 & 3 & 4 & 5 & 6 & 7 \\
\hline \multicolumn{8}{|l|}{ FAMÍLIA } \\
\hline 37. Tenho um convívio bom com a minha família. & 0,84 & & & & & & \\
\hline 25. Minha família me faz feliz. & 0,84 & & & & & & \\
\hline 51. Eu me divirto com a minha família. & 0,80 & & & & & & \\
\hline 58. Meus pais são carinhosos comigo. & 0,78 & & & & & & \\
\hline 13. Minha família se dá bem. & 0,74 & & & & & & \\
\hline 73. Minha família gosta de mim. & 0,73 & & & & & & \\
\hline 63. Gostaria que minha família fosse diferente. & 0,71 & & & & & & \\
\hline 80. Minha família me ajuda quando preciso. & 0,70 & & & & & & \\
\hline 5. Sou compreendido em casa. & 0,61 & & & & & & \\
\hline 7. Eu fico feliz quando a minha família se reúne. & 0,53 & & & & & & \\
\hline \multicolumn{8}{|l|}{ SELF } \\
\hline 79. Eu sou alegre. & 0,31 & 0,74 & & & & & \\
\hline 43. Eu sou uma pessoa bem humorada. & & 0,75 & & & & & \\
\hline 17. Eu sorrio bastante. & & 0,72 & & & & & \\
\hline 57. Eu sou divertido. & & 0,69 & & & & & \\
\hline 71. Eu me considero uma pessoa descontraída. & & 0,61 & & & & & \\
\hline 53. Eu sou feliz. & & 0,57 & & & & & \\
\hline 1. Eu me divirto com muitas coisas. & & 0,53 & & & & & \\
\hline 83. Gosto da minha vida. & & 0,46 & & & & & \\
\hline 62. Eu me sinto bem do jeito que sou. & & 0,36 & & & & & 0,30 \\
\hline \multicolumn{8}{|l|}{ ESCOLA } \\
\hline 60. Eu gosto de ir à escola. & & & 0,80 & & & & \\
\hline 67. Eu me sinto bem na minha escola. & & & 0,75 & & & & \\
\hline 29. Eu me divirto na escola. & & & 0,73 & & & & \\
\hline 47. Meus professores são legais comigo. & & & 0,70 & & & & \\
\hline 16. Eu gosto das atividades da escola. & & & 0,66 & & & & \\
\hline 70. Eu aprendo muitas coisas na escola. & & & 0,66 & & & 0,30 & \\
\hline \multicolumn{8}{|l|}{ SELF COMPARADO } \\
\hline 77. Meus amigos se divertem mais do que eu. & & & & 0,79 & & & \\
\hline 10. Meus amigos passeiam mais do que eu. & & & & 0,77 & & & \\
\hline 28. Meus amigos podem fazer mais coisas do que eu. & & & & 0,75 & & & \\
\hline 45. Outros adolescentes ganham mais presentes do que eu. & & & & 0,68 & & & \\
\hline 2. Os outros adolescentes têm mais amigos do que eu. & & & & 0,61 & & & \\
\hline 49. Os outros adolescentes são mais alegres do que eu. & & & & 0,42 & & & \\
\hline \multicolumn{8}{|l|}{ NÃO-VIOLÊNCIA } \\
\hline 65. Sou irritado. & & & & & 0,78 & & \\
\hline 24. Mantenho a calma. & & & & & 0,66 & 0,35 & \\
\hline 50. Gosto de brigas. & & & & & 0,58 & & \\
\hline 32. Brigar resolve problemas. & & & & & 0,56 & & \\
\hline 54. Eu me sinto calmo, tranqüilo. & & & & & 0,56 & & \\
\hline 19. Brigo muito com meus amigos. & & & & & 0,42 & 0,31 & \\
\hline \multicolumn{8}{|l|}{ AUTO-EFICÁCIA } \\
\hline 41. Tenho sucesso em atividades que realizo. & & & & & & 0,63 & \\
\hline 82. Sou capaz de realizar muitas coisas. & & & & & & 0,53 & \\
\hline 8. Vou atrás do que quero conquistar. & & & & & & 0,52 & \\
\hline 48. Eu sou inteligente. & & & & & & 0,50 & \\
\hline 38. Faço o que gosto de fazer. & 0,35 & & & & & 0,43 & \\
\hline 31. É difícil conseguir o que quero. & & & & 0,32 & & 0,40 & \\
\hline 30. Consigo expressar minhas idéias. & & & & & & 0,34 & \\
\hline \multicolumn{8}{|l|}{ AMIZADE } \\
\hline 35. É bom sair com meus amigos. & & & & & & & 0,72 \\
\hline 52. Gosto de conversar com meus amigos. & & & & & & & 0,71 \\
\hline 21. Eu me divirto com meus amigos. & & & & & & & 0,67 \\
\hline 26. Eu gostaria que meus amigos fossem diferentes. & & & & & 0,36 & & 0,61 \\
\hline 11. Eu me relaciono bem com meus amigos. & 0,31 & & & & & & 0,58 \\
\hline 72. Meus amigos me ajudam quando preciso. & & & & & & & 0,52 \\
\hline 66. Meus amigos gostam de mim. & 0,36 & & & & & & 0,50 \\
\hline 76. Gosto de sair para me divertir. & 0,33 & & & & & & 0,48 \\
\hline
\end{tabular}

Nota: os itens com sentido negativo tiveram seus escores revertidos antes da análise. Os números dos itens na tabela correspondem à numeração original, antes da exclusão de itens. Cargas fatoriais inferiores a 0,30 foram suprimidas. 
satisfação desses relacionamentos, algumas indicações de lazer e situações de diversão e apoio.

A Tabela 1 apresenta a matriz de componentes da ESVMA, com as cargas componenciais, os autovalores e a variância explicada por cada um dos componentes. Cabe ressaltar que a versão final explicou $54,0 \%$ da variância total encontrada nesta amostra.

Foram calculados escores para cada uma das subescalas da ESVMA computando-se a média dos itens correspondentes em cada dimensão. Ressalta-se que o escore de cada uma das variáveis varia de 1 a 5 , sendo que a média foi calculada por meio do somatório total dividido pelo número de itens, uma vez que cada subescala apresenta diferenças na quantidade dos itens que a compõem.

As subescalas apresentaram níveis de fidedignidade (alfa de Cronbach) satisfatórios, variando entre 0,70 a 0,91, sendo que para a escala total este valor foi 0,93 . O número de itens, a média, o desvio-padrão de cada dimensão, bem como os níveis de fidedignidade podem ser observados na Tabela 2.

Foi realizada uma análise de variância multivariada (MANOVA) $2 \times 2 \times 2$ para investigar os efeitos do sexo, do tipo de escola e da faixa etária, tendo como variáveis dependentes as sete subescalas. Esta análise revelou um efeito principal significativo para sexo [Lambda de Wilks $(7,409)=0,935$; $\mathrm{p}<0,001]$ e uma interação significativa entre faixa etária e tipo de Escola [Lambda de Wilks $(7,409)=0,966 ; \mathrm{p}<0,05]$. Análises univariadas indicaram níveis de satisfação maiores nos adolescentes do sexo masculino nos domínios Família $[\mathrm{F}(1,415)=5,13 ; \mathrm{p}=0,02]$, Self Comparado $[\mathrm{F}(1,415)=5,79$; $\mathrm{p}=0,02]$, Não-violência $[\mathrm{F}(1,415)=10,16$; $\mathrm{p}<0,01]$ e Auto-eficácia $[\mathrm{F}(1,415)=6,49 ; \mathrm{p}=0,01]$. Já as adolescentes mulheres tenderam a apresentar um maior nível de satisfação no domínio Escola, embora a diferença tenha sido apenas marginalmente significativa $[\mathrm{F}(1,415)=2,99 ; \mathrm{p}=0,08]$.

As análises univariadas conduzidas para especificar melhor a interação observada também revelaram efeitos marginalmente significativos, e apenas para duas variáveis: Self $[\mathrm{F}(1,415)=2,92 ; \mathrm{p}=0,09]$ e Escola $[\mathrm{F}(1,415)=3,46 ; \mathrm{p}=0,06]$. Em caráter exploratório, testes t complementares foram realizados. Essas análises mostraram que os adolescentes de escola pública na faixa de 17 a 19 anos apresentaram escores mais baixos do que os da faixa etária de 14 a 16 anos nas dimensões Self $[\mathrm{t}(215)=2,05 ; \mathrm{p}=0,04]$ e Escola $[\mathrm{t}(215)=3,07$; $\mathrm{p}<0,01]$. Além disso, observou-se uma diferença entre a escola pública e a particular na dimensão Self (em favor da escola particular), mas apenas na faixa etária de 17 a 19 anos $[\mathrm{t}(161)=2,45 ; \mathrm{p}=0,02]$.

Tabela 2. Propriedades das Dimensões e da Escala Total Multidimensional de Satisfação de Vida para Adolescentes

\begin{tabular}{lcccc}
\hline & $\mathrm{N}^{\circ}$ Itens & Média & DP & Alfa \\
\hline 1. Família & 10 & 4,05 & 0,27 & 0,91 \\
2. Self & 09 & 4,07 & 0,19 & 0,87 \\
3. Escola & 06 & 3,20 & 0,34 & 0,85 \\
4. Self Comparado & 06 & 3,73 & 0,20 & 0,85 \\
5. Não-violência & 06 & 3,90 & 0,64 & 0,70 \\
6. Auto-eficácia & 07 & 3,80 & 0,25 & 0,71 \\
7. Amizade & 08 & 4,23 & 0,28 & 0,82 \\
Escala Total & 52 & 3,85 & 0,31 & 0,93 \\
\hline
\end{tabular}

A Tabela 3 exibe as correlações entre as subescalas da EMSVA. Com exceção das subescalas Self Comparado e Escola, foram encontradas correlações significativas entre as dimensões do instrumento.

Com o intuito de obter evidências complementares de validade para a EMSVA, calcularam-se as correlações de cada subescala com a variável auto-estima. Foram observados os seguintes valores de correlação ( $\mathrm{r}$ de Pearson) entre auto-estima e cada subescala: Família $(\mathrm{r}=0,34 ; \mathrm{p}<0,01)$, Self $(\mathrm{r}=0,51 ; \mathrm{p}<0,01)$, Escola $(\mathrm{r}=0,06 ; \mathrm{p}=0,25)$, Self Comparado $(\mathrm{r}=0,41 ; \mathrm{p}<0,01)$, Não-violência $(\mathrm{r}=0,29 ; \mathrm{p}<0,01)$, Auto-eficácia $(\mathrm{r}=0,56 ; \mathrm{p}<0,01)$ e Amizade $(\mathrm{r}=0,32 ; \mathrm{p}<0,01)$.

\section{Discussão}

O estudo teve por objetivo desenvolver a Escala Multidimensional de Satisfação de Vida para Adolescentes (EMSVA) e obter evidências de sua estrutura dimensional e fidedignidade. As análises iniciais da EMSVA identificaram dimensões coerentes e psicologicamente relevantes, embora a estrutura final não tenha confirmado todos os domínios previamente estabelecidos. Além disso, encontraram-se índices de consistência interna adequados para cada uma das subescalas, assim como para a escala total.

Apesar da escala ter apresentado propriedades psicométricas satisfatórias, é necessária uma discussão sobre quais dimensões deveriam compor uma escala de satisfação de vida para adolescentes. As dimensões presentes na EMSVA foram baseadas em evidências empíricas, obtidas por meio de entrevistas com os adolescentes e outros instrumentos que avaliam satisfação de vida. Contudo, a continuidade das investigações é necessária para determinação da pertinência das dimensões propostas neste estudo.

As sete dimensões identificadas (Família, Self, Escola, Self Comparado, Não-violência, Auto-eficácia e Amizade) possuem sentido teórico e confirmam quatro dos cinco domínios propostos por Huebner (1994). Foi verificada a manutenção dos dois domínios acrescentados nos estudos de Giacomoni e Hutz (2008) para satisfação de vida na infância: Self Comparado e Não-violência. A permanência do domínio Self Comparado indica a importância das teorias de comparação social para a compreensão da satisfação de vida em adolescentes. A relevância dessa abordagem já havia sido apontada em estudos com crianças (Giacomoni \& Hutz, 2008) e adultos (Wood, 1996; Diener, Suh, Lucas \& Smith, 1999).

Tabela 3. Matriz de Correlação entre as subescalas da EMSVA

\begin{tabular}{|c|c|c|c|c|c|c|}
\hline & 1 & 2 & 3 & 4 & 5 & 6 \\
\hline 1. Família & - & & & & & \\
\hline 2. Self & $0,44^{*}$ & & & & & \\
\hline 3. Escola & $0,27 *$ & $0,21 *$ & & & & \\
\hline 4. Self Comparado & $0,34 *$ & $0,42 *$ & 0,08 & & & \\
\hline 5. Não-violência & $0,36^{*}$ & $0,32 *$ & $0,22 *$ & $0,19 *$ & & \\
\hline 6. Auto-eficácia & $0,45^{*}$ & $0,53 *$ & $0,30 *$ & $0,43 *$ & $0,27 *$ & \\
\hline 7. Amizade & $0,31 *$ & $0,56^{*}$ & $0,15^{*}$ & $0,45^{*}$ & $0,19 *$ & $0,39 *$ \\
\hline
\end{tabular}


Destaca-se no modelo final da EMSVA o fato da primeira dimensão ser composta pelos itens referentes à família. $\mathrm{O}$ modelo distingue-se do presente na Escala Multidimensional de Satisfação de Vida de Crianças (Giacomoni \& Hutz, 2008), na qual a primeira dimensão foi Self, seguida de Família. Contrariando perspectivas desenvolvimentais que apontam um afastamento do adolescente de seu núcleo familiar, os resultados da estrutura da EMSVA reforçam o papel da família no desenvolvimento positivo do bem-estar subjetivo durante a adolescência.

Apesar do aumento da complexidade das relações familiares na adolescência, ter uma família unida encontra-se entre uma das necessidades mais relevantes para os adolescentes considerarem-se felizes (Zagury, 2003). Outros autores argumentam ainda que, para o núcleo familiar servir como fonte de apoio social para o desenvolvimento saudável do adolescente, características positivas como a presença de relações afetivas, coesão, segurança e ausência de discórdias deveriam estar presentes (Steinberg, 1999).

A identificação da dimensão Auto-eficácia, por sua vez, sugere a importância que a existência de metas e a percepção de esforços pessoais bem sucedidos têm para a satisfação com a vida durante a adolescência. Tal influência já foi ressaltada por outros autores (Cantor, 1994; Cantor \& Sanderson, 1999; Emmons, 1986; Diener \& Lucas, 1999) e, apesar de correlações entre medidas de auto-eficácia e satisfação de vida serem encontradas na literatura (Ronen \& Seeman, 2007), propostas de inclusão desse construto como um fator em escalas de avaliação de bem-estar eram inexistentes. Assim, mais estudos são necessários para a verificação da pertinência dessa dimensão em modelos de satisfação de vida na adolescência.

Dentre todas as subescalas, os adolescentes obtiveram a média mais alta no fator referente às amizades. $\mathrm{O}$ resultado reforça a indicação da literatura de que a qualidade das experiências com pares vivenciadas durante a adolescência é uma dimensão importante na avaliação da satisfação de vida nessa etapa da vida (Huebner, Drane e Valois, 2000). No geral, os adolescentes que participaram deste estudo apresentaram boas médias de satisfação de vida em todas as subescalas (acima do ponto médio das escalas). Pesquisas demonstram que, independentemente de configuração familiar, classe econômica, gênero e escolaridade, os adolescentes tendem a apresentar bons níveis em medidas globais (Diener, 1996; Wagner, Ribeiro, Arteche \& Bornholdt, 1999) e multidimensionais de satisfação de vida (Huebner, Drane \& Valois, 2000).

Com relação à idade, pouco se conhece seu efeito sobre a satisfação de vida em adolescentes. Enquanto as pesquisas de Huebner $(1994,1991 c)$ apontam uma estabilidade, neste estudo, observou-se uma tendência para escores menores de satisfação nas escolas públicas, mais especificamente na faixa de 17 a 19 anos. É interessante observar também que a menor média geral de satisfação foi verificada no domínio Escola. Huebner, Drane e Valois (2000) obtiveram resultados semelhantes e atentaram para as experiências escolares dos adolescentes americanos estarem servindo como fonte de insatisfação e estresse. Níveis baixos de satisfação com o mesmo domínio também foram encontrados por Huebner (2004) e geraram questionamentos relacionados ao papel das escolas na educação e promoção de saúde dos adolescentes. Como avaliado por Marriel, Assis, Avanci e Oliveira (2006) a escola é um espaço privilegiado para o desenvolvimento saudável do adolescente, sendo assim, futuras investigações são necessárias para se encontrar determinantes e correlatos da satisfação dos adolescentes nesse importante domínio da vida.

Os achados referentes à variável sexo são ainda inconsistentes na literatura sobre satisfação de vida de adolescentes. Enquanto nos estudos de Huebner (1991a, 1991b, 1991c) não se encontraram diferenças de sexo quanto à satisfação de vida em adolescentes, outras pesquisas evidenciaram níveis de satisfação de vida desiguais para meninos e meninas (Degode, Spruijt \& Maas, 1999; Huebner, Drane \& Valois, 2000)

Indicações da validade concorrente das subescalas da EMSVA foram confirmadas por correlações modestas com a medida de auto-estima. Esse resultado indica que existe certa tendência dos adolescentes com escores altos em auto-estima também apresentarem bons escores de satisfação de vida nos diferentes domínios avaliados, em concordância com a premissa de que uma visão positiva de si mesmo pode promover benefícios e predizer índices de satisfação com a vida e bem-estar subjetivo (Heatherton \& Wyland, 2005). As correlações positivas de medidas de satisfação de vida e auto-estima encontradas também concordam com estudos de validade concorrente desses construtos realizados em outros países (Dew \& Huebner, 1994; Huebner, Drane \& Valois, 2000).

Em síntese, os resultados deste estudo sugerem que a EMSVA apresenta evidências de validade quanto à sua estrutura dimensional, validade concorrente com a variável auto-estima, além de boa fidedignidade, o que encoraja o uso da escala em novas pesquisas. Mais estudos que avaliem a validade do instrumento, porém, são necessários. Por exemplo, é preciso investigar se a escala possui poder discriminante entre diferentes grupos com características diferenciadas. Além disso, deve-se considerar que a amostra utilizada neste estudo foi restrita a uma cidade no sul do país, sendo assim, os resultados não podem ser generalizados para outros contextos. De qualquer modo, espera-se que a EMSVA possa servir de estímulo para o avanço das pesquisas no campo da psicologia positiva entre jovens.

\section{Referências}

Albuquerque, A. S., \& Troccóli, B. T. (2004). Desenvolvimento de um escala de bem-estar subjetivo. Psicologia: Teoria e Pesquisa, 20(2), 153-164.

Arteche, A. X., \& Bandeira, D. R. (2003). Bem-estar subjetivo: um estudo com adolescentes trabalhadores. Psico USF, 8, 193-201.

Assis, S. G., Avanci, J. Q., Silva, C. M. F. P., Malaquias, J. V., Santos, N. C., \& Oliveira, R. V. C. (2003). A representação social do ser adolescente: um passo decisivo na promoção da saúde. Ciência \& Saúde Coletiva, 8(3), 669-680.

Ben-Zur, H. (2003). Happy adolescents: the link between subjective well-being, internal resources, and parental factors. Journal of Youth and Adolescence, 32(2), 67-79.

Cantor, N. (1994). Life task problem solving: situation affordances and personal needs. Personality and Social Psychology Bulletin, 20, 235-43. 
Cantor, N., \& Sanderson, C. (1999). Life task participation and well-being: The importance of taking part in daily life. Em D. Kahneman, E. Diener, \& N. Schwarz (Eds.), Well-being: The foundations of hedonic psychology (pp. 230-243). New York: Russel Sage Foudation.

Dancey, C. P., \& Reidy, J. (2006). Estatística sem matemática. (L. Viali, Trad.). Porto Alegre: Artmed.

Degode, M., Spruijt, E., \& Maas, C. (1999). Individual and family factors and adolescent well-being: A multi-level analysis. Social Behavior and Personality, 27(3), 263-280.

Dew, T., \& Huebner, E. (1994). Adolescents perceived quality of life: An exploratory investigation. Journal of School Psychology, 32, 185-199.

Diener, E. (1984). Subjective well-being. Psychological Bulletin, 95, 542-575.

Diener, E. (1996). Subjective well-being in cross-cultural perspective. Em H. Grad (Ed.) Key issues in cross-cultural psychology: Selected papers from the Twelfth International Congress of the International Association for Cross-Cultural Psychology (pp.319-331). Champaign-Urbana, IL.

Diener, E., \& Lucas, R. E. (1999). Personality and Subjective wellbeing. Em D. Kahneman, E., Diener, \& N. Schwarz (Eds.), Wellbeing: The foundations of hedonic psychology. (pp. 213-229). New York: Russel Sage Foudation.

Diener, E., Suh, E. M., Lucas, R. E., \& Smith, H. L. (1999). Subjective well-being: Three decades of Progress. Psychological Bulletin, 125, 276-302.

Emmons, R. A. (1986). Personal strivings: an approach to personality and subjective well-being. Journal of Personality and Social Psychology, 51, 158-168.

Giacomoni, C. H., Höher, S. P., Wathier, J. L., Santos, B. R., Segabinazi, J. D., \& D'ávila, V. S. (2005). Avaliação Qualitativa de Bem-Estar Subjetivo em Adolescentes [Resumos]. Em Instituto Brasileiro de Avaliação Psicológica (Org.). Anais II Congresso Brasileiro de Avaliação Psicológica. (s/p). Gramado, RS.

Giacomoni, C. H., \& Hutz, C. S. (2008). Escala Multidimensional de Satisfação de Vida para Crianças: estudos de construção e validação. Revista Estudos de Psicologia, 25(1), 23-35.

Gilman, R., Huebner, E. S., \& Laughlin, J. E. (2000). A first study of the multidimensional students' life satisfaction scale. Social Indicators Research, 52, 135-160.

Heatherton, T. F., \& Wyland, C. L. (2005). Assessing Self-Esteem. Em S. J. Lopez, \& C. R. Snyder (Eds.), Positive Psychological Assessment: A Handbook of Models and Measures, 14, (pp. 219-233). Washington: APA.

Huebner, E. S. (1991a). Initial development of the student's life satisfaction scale. School Psychology International, 12(3), 229-238.

Huebner, E. S. (1991b.) Correlates of life satisfaction in children. School Psychology Quarterly, 6(2), 103-111.

Huebner, E. S. (1991c). Further validation of the students' life satisfaction scale: The independence of satisfaction and affect ratings. Journal of Psychoeducational Assessment, 9, 363-368.

Huebner, E. S. (1994). Preliminary development and validation of a multidimensional life satisfaction scale for children. Psychological Assessment, 6(2), 149-158.

Huebner, E. S. (1998a). Cross-racial application of a children's multidimensional life satisfaction scale. School Psychology International, 19(2), 179-188.
Huebner, E. S. (1998b). “Cross-racial application of a children's multidimensional life satisfaction scale": Erratum. School Psychology International, 19(4), 290.

Huebner, E. S. (2004). Research on assessment of life satisfaction on children and adolescents. Social Indicators Research, 66, 3-33.

Huebner, E. S., Drane, W., \& Valois, R. F. (2000). Levels and demographic correlates of adolescence life satisfaction reports. School Psychology International, 21, 281-292.

Huebner, E. S., \& Gilman, R. (2002). An introduction to the multidimensional students' life satisfaction scale. Social Indicators Research, 60, 115-122.

Huebner, E. S., Gilman, R., \& Laughlin, J. E. (1999). A multimethod investigation of the multidimensionality of children's wellbeing reports: Discriminant validity of life satisfaction and Self-esteem. Social Indicators Research, 46, 1-22.

Huebner, E. S., Laughlin, J. E., Ash, C., \& Gilman, R. (1998). Further validation of the multidimensional students' life satisfaction scale. Journal of Psychoeducational Assessment, 16, 118-134.

Hunter, J P. \& Csikszentmihalyi, M. (2003). The positive psychology of interested adolescents. Journal of Youth and Adolescence, $32(1), 27-35$.

Hutz, C. S. (2000). Adaptação brasileira da escala de auto-estima de Rosenberg. Manuscrito não publicado. Universidade Federal do Rio Grande do Sul. Porto Alegre, RS.

Marriel, L. C., Assis, S. G., Avanci, J. Q., \& Oliveira, R. V. C. (2006). Violência escolar e auto-estima de adolescentes. Cadernos de Pesquisa, 36(127), 35-50.

Michalos, A. C. (1985). Multiple discrepancies theory (MDT). Social Indicators Research, 16, 347-413.

Ronen, T., \& Seeman, A. (2007). Subjective well being of adolescents in boarding schools under threat of war. Journal of Traumatic Stress, 20(6), 1053-1062.

Rosenberg, M. (1965). Society and the adolescent Self-image. Princeton: Princeton University Press.

Seligman, M. E. P., \& Csikszentmihalyi, M. (2000). Positive Psychology: An Introduction. American Psychology, 55, 5-14.

Steinberg, L. (1999). Adolescence. Boston: McGraw-Hill.

Suldo, S. M., \& Huebner, E. S. (2006). Is extremely high life satisfaction during adolescence advantageous? Social Indicators Research, 78, 179-203.

Terry, T., \& Huebner, E. S. (1995). The relationship between Selfconcept and life satisfaction in children. Social Indicators Research, 35, 29-52.

Wagner, A., Ribeiro, L., Arteche, A., \& Bornholdt, E. (1999). Configuração familiar e o bem-estar psicológico dos adolescentes. Psicologia: Reflexão e Crítica, 12(1), 147-156.

Wood, J. V. (1996). What is social comparison and how should we study it? Personality and Social Psychology Bulletin, 22, 520-537.

Zagury, T. (2003). O adolescente e a felicidade. Ciência e Saúde Coletiva, (8)3, 681-689.

Recebido em 23.01.09

Primeira decisão editorial em 29.06.09

Versão final em 23.09.09

Aceito em 30.09.09 